\title{
Evidence-Based Practices for the Identification, Screening, and Prevention of Acute Delirium in the Hospitalized Elderly: An Overview of Systematic Reviews
}

\author{
Cheryl Holly • E. Renee Cantwell • Mary C. Kamienski
}

Published online: 16 December 2012

(C) Springer Science+Business Media New York 2012

\begin{abstract}
This paper presents an overview of systematic reviews related to the identification and prevention of acute delirium in the hospitalized elderly. The need to build an evidence base in these areas is heightened by reports that delirium is associated with high rates of readmission, and increases in mortality, length of stay, and cost. We searched MEDLINE, CINAHL, Academic Search Premiere, the Cochrane Database of Systematic Reviews (CDSR) and the Joanna Briggs Institute Library of Systematic Reviews. The search strategy was designed to find systematic reviews, a form of secondary research that synthesizes findings from primary studies in an attempt to identify best practice. A total of 13 systematic reviews were retrieved. Of these, seven were focused on risk factors, three on screening, and three on prevention strategies. Recommendations related to best practice for the identification, screening and prevention of delirium in hospitalized patients over 65 years are provided.
\end{abstract}

Keywords Delirium · Hospital · Acute confusion · ICU psychosis $\cdot$ Elderly $\cdot$ Risk factors $\cdot$ Screening and prevention

\section{Introduction}

Every day elderly adults are admitted to hospitals with acute illness or injury. Of these, up to $42 \%$ of elderly medical patients and $80 \%$ of elderly critical care patients experience delirium [1-3], which often goes unrecognized [4]. The preferred definition of delirium from the Diagnostic and

C. Holly $(\bowtie) \cdot$ E. R. Cantwell $\cdot$ M. C. Kamienski

University of Medicine and Dentistry of New Jersey School of

Nursing, 65 Bergen Street, Suite 1127,

Newark, NJ 07101, USA

e-mail: hollych@umdnj.edu
Statistical Manual of Mental Disorders (DSM - IV) states "delirium is a disturbance of consciousness that is accompanied by a change that cannot be better accounted for by a pre-existing or evolving dementia" [5]. Delirium is characterized by acute onset, altered level of consciousness, and a changeable course of disturbance in orientation, memory, attention, thought, and behavior [6]. Delirium has recently been reported to be among the top six preventable conditions among hospitalized patients older than 65 years [7]. Delirium is known to develop in three types [2], but its precise pathology is unknown. "The most commonly accepted premise is a neurotransmitter abnormality with cholinergic deficiency that affects multiple spheres of the central nervous system, although an undetected diffuse brain injury has also been implicated" [2, p.137]. This paper presents an overview of evidence generated by systematic reviews on risk factors for delirium, the use of screening tools, and prevention strategies. The need to build an evidence base in these areas is accentuated by reports that delirium is associated with high rates of 30- to 90-day readmissions [8], and increases in mortality [9], length of stay [10], and cost [11].

Papers published from 2000 through October 2012 using the keywords: delirium, hospital, acute confusion, ICU psychosis, elderly, risk factors, screening and prevention were sought. We searched MEDLINE, CINAHL, Academic Search Premiere, the Cochrane Database of Systematic Reviews (CDSR) and the Joanna Briggs Institute Library of Systematic Reviews for relevant articles. Specifically, the search strategy was designed to find systematic reviews, a form of secondary research that synthesizes findings from primary studies in an attempt to identify best practice. A total of 13 systematic reviews were retrieved. Of these, seven were focused on risk factors, three on screening, and three on prevention strategies. The majority of these were published between 2006 and 2012 . 


\section{The Evidence on Risk Factors for Acute Delirium}

According to the World Health Organization, a risk factor is any "attribute, characteristic or exposure of an individual that increases the likelihood of developing a disease or injury" [12]. For example, smoking is a risk factor for heart disease. Multiple risk factors are implicated in the development of delirium, including the use of some medications, the environment, and invasive procedures. In addition, there are biomarkers that can determine the potential for onset of acute delirium in elders. In an early review, Elie and colleagues [13] reported that certain risk factors for delirium were consistent among patients. Included were dementia, advanced age, and medical illness. They reported that male gender, depression, alcohol abuse, hearing impairment and decreased functional status were important indicators. Since this early study, published in 1998, seven other systematic reviews on risk factors were located; all were published between 2006 and 2012. The findings of these six reviews were re-synthesized into risk categories related to pharmacology, setting, procedure, and biomarkers (See Table 1).

Pharmacological Risk Polypharmacy, the administration of multiple medications some of which may be unnecessary, is known to contribute to untoward outcomes in the older adult, any of which may be aggravated upon hospital admission. Mattar et al. [14••] and Van Rampaey et al. [15] found evidence to support this observation in their systematic reviews. Mattar et al. [14••] identified benzodiazepines as the most significant drug causing delirium in ICU patients. Benzodiazepines (e.g., Ativan) increase the effect of the neurotransmitter GABA, resulting in increased sedation and hypnosis (sleep) with symptoms manifesting as delirium. Van Rompaey et al. [15] found that the odds ratio (OR) was highest for development of delirium in patients receiving an intermediate daily morphine dose between 7.2 and $18.6 \mathrm{mg}$. The administration of lorazepam and dopamine also were associated with higher risk $[15,16]$.

Environmental Risk In a review of 24 cohort studies (two retrospective; 22 prospective) comprising over 1,000 subjects, Mattar et al., [14・•] examined the relationship between setting and the development of delirium. They concluded that, while older age and the presence of co-morbidities (history of stroke and dementia) were similar across hospital units, patients in different areas of the hospital have different risk factors for acute delirium. For example, the presence of sepsis and heavy alcohol use were the most significant risk factors in the medical ICU. In the surgical ICU, hypoalbuminemia, impaired functional status, high APACHE (Acute Physiology and Chronic Health Evaluation) II score and high serum cortisol level were associated with elevated risk of delirium. A high APACHE II score was the most significant risk factor. The APACHE measures severity of illness and risk of death, suggesting a relationship between physiological stress and the development of delirium. Among patients in trauma units, those with low Glascow Coma Scores and multiple blood transfusions were found to have a higher risk of acute delirium. In the cardiac ICU, the risk factors shown for delirium included the following: increased duration of cardiac surgery (particularly, valve surgery with or without coronary artery bypass grafting $(\{\mathrm{CABG}\})$, prolonged intubation time, low intraoperative body temperature, peripheral vascular disease, preoperative intra-arterial blood pressure support, blood product usage, and low cardiac output during the postoperative period. Patients admitted with acute myocardial infarction following a cardiac arrest who exhibited high blood levels of potassium were also more likely to develop delirium. A more recent review by Koster et al. [17], also found an association between cardiac surgery and the development of delirium.

Van Rompaey et al. [15] studied the occurrence of delirium in intensive care units. The review encompassed six studies (five prospective cohort studies; one chart review) and included 5,434 patients. The reviewers divided their findings into predisposing and precipitating factors. Among the predisposing factors, the one exhibiting the highest odds ratio was history of respiratory disease. Among the 21 precipitating factors, the APACHE II score and hypertension were identified as significant predictors of risk.

Postoperatively, Fong [18] found that administration of meperidine was consistently associated with an increased risk of delirium in elderly surgical patients, but there was no evidence to demonstrate a relationship with more frequently used postoperative opioids, such as morphine or fentanyl.

Procedural Risk In a review of 19,068 patients over ten cohort studies (three prospective; seven retrospective) using three databases, Koster et al. [17] found cognitive impairment, a history of stroke, presence of depression, and older age (over 65 years) to be frequently identified in patients experiencing delirium following cardiac surgery. A wide range in reported odds ratios and large confidence intervals were found in the majority of studies reviewed. The reviewers determined that it was not possible to state whether any of the risk factors were true independent causal variables or only markers that reflected the general medical condition of the patient, concluding that the clinical pathway to delirium is at present unknown. However, they did posit that a risk model using multiple predisposing and precipitating factors should be developed so that a checklist for risk of acute delirium can be developed. A review by Dasgupta et al. [19] of 25 studies, of which 21 were isolated risk factor studies, three were validation studies only, and one was both a validation and risk factor study, found there was 


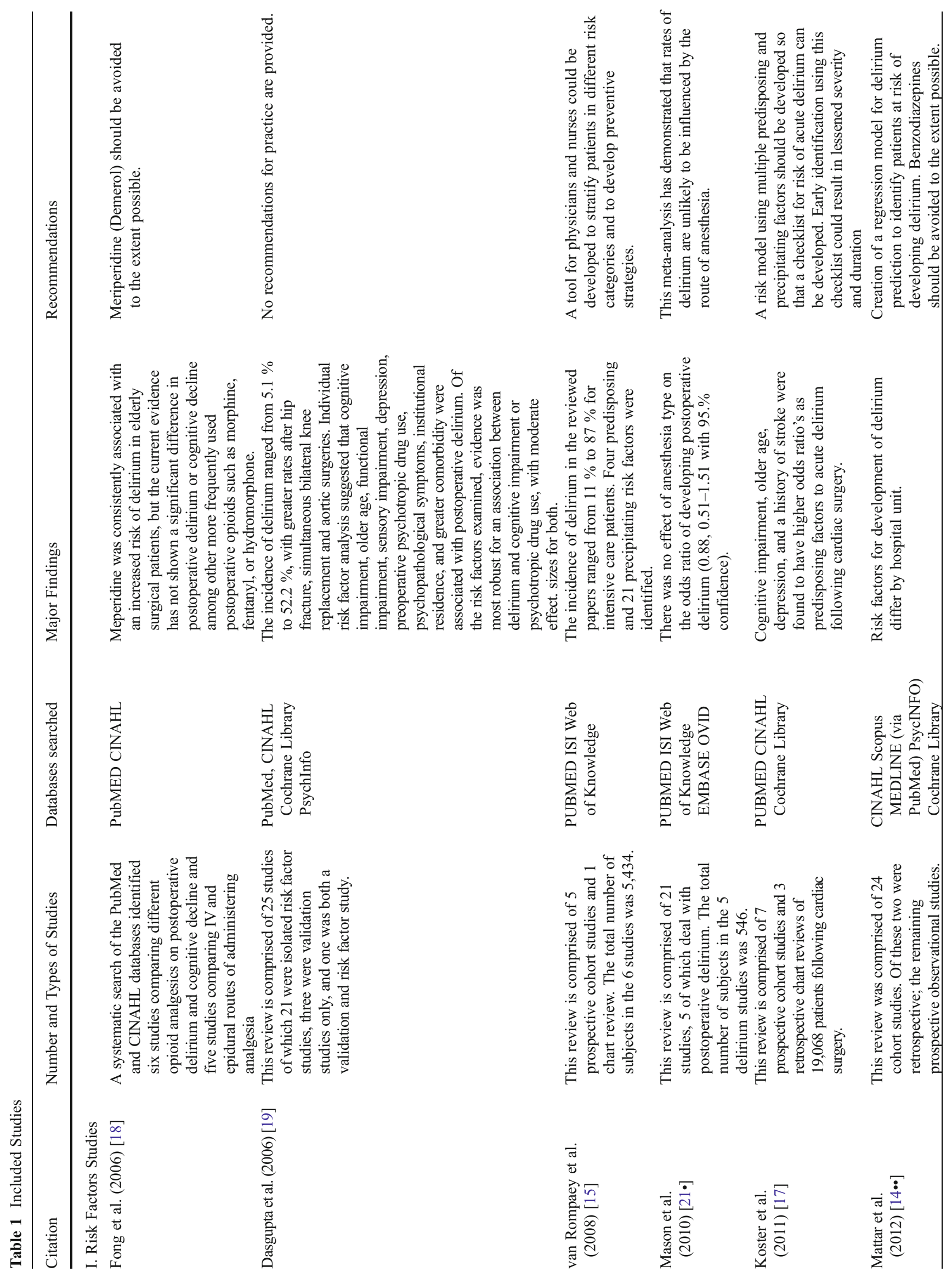




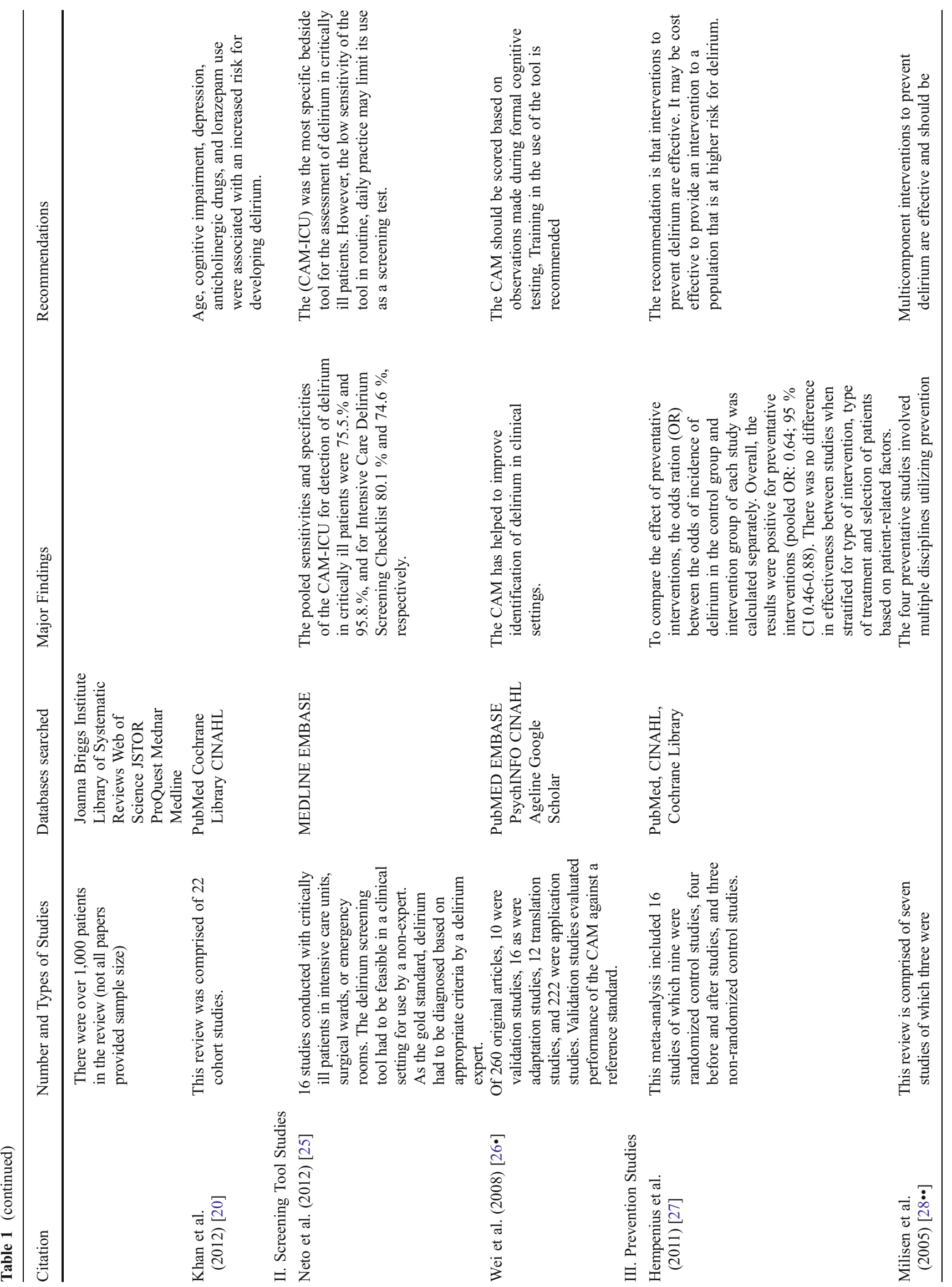




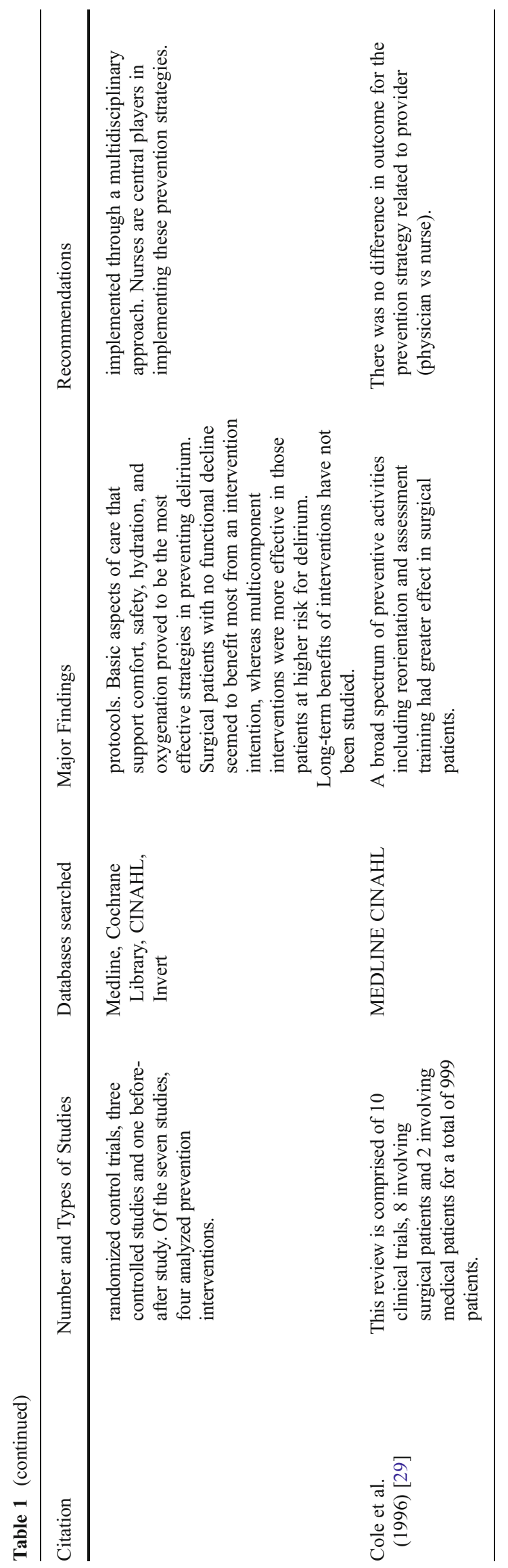

insufficient evidence to support an association between male sex and delirium as reported earlier by Elie et al. [13)] which had a smaller number of female participants possibly accounting for the discrepancy in results. In Dasgupta, et al.'s review [19], cognitive impairment was consistently associated with delirium and had moderate effect sizes in pooled analyses, which suggests that cognitive impairment and psychotropic drug use are the risk factors most robustly associated with delirium, given their moderate range of effect sizes. Over the 25 studies, the incidence of delirium ranged from $5.1 \%$ to $52.2 \%$, with greater rates after hip fracture, simultaneous bilateral knee replacement and aortic surgeries. Analysis of individual risk factors suggested that each of the following was associated with postoperative delirium: cognitive impairment, older age, functional impairment, sensory impairment, depression, preoperative psychotropic drug use, psychopathological symptoms, institutional residence, and greater comorbidity. Of the risk factors examined, evidence was most robust for associations among delirium, and cognitive impairment and psychotropic drug use, with moderate effect sizes for both. These findings are similar to those of Kahn et al. [20] in a review of 22 studies in which age, cognitive impairment, depression, anticholinergic drugs, and lorazepam use were associated with an increased risk for the development of delirium.

Mason, et al. [21•] aimed to determine the impact of the route of anesthetic delivery on the development of postoperative delirium (POD). There was no effect of anesthesia type on the odds ratio of developing POD $(0.88,0.51-1.51$ with $95 \%$ confidence). They concluded that general anesthesia has not been shown to influence development of POD.

Biomarker Risk Lower tryptophan levels, higher plasma cortisol, higher interleukin - 6 levels, higher CRP levels, anemia, hypocalcaemia, hyponatremia, azotemia, elevated liver enzymes, hyperamylasemia, hyperbilirubinemia and metabolic acidosis were all found to be significant risk factors for delirium [14*•]. In the intensive care unit, hyperamylasaemia, hypocalcaemia, hyperbilirubiemia and hyponatremia were reported as the strongest risk factors [15].

Over all, this literature supports the conclusion that delirium is a disorder with multiple risk factors. Systematic review consistently demonstrated that those aged 65 years and older, with a pre-existing cognitive impairment (including depression), psychotropic or opioid drug administration, and having a surgical procedure (often orthopedic or cardiac) were at elevated risk for the development of delirium. Others at risk include severely ill intensive care unit patients with high APACHE II scores and a variety of abnormal blood values, particularly hypoalbuminenmia and hyponatremia. These findings highlight the need for development of a risk model that uses predisposing and precipitating factors to construct a checklist for early identification. 


\section{The Evidence on Use of Screening Tools for Acute Delirium}

Evaluation for delirium replaces neither clinical judgment nor formal cognitive screening measures; rather, it complements them by providing additional information, which may help to identify subtle or atypical markers of delirium [16]. Typically, delirium is diagnosed by psychiatric consultation using the Diagnostic and Statistical Manual (DSM). New screening tools such as the Confusion Assessment Method (CAM), and its modified version for the Intensive Care Unit (CAM - ICU) and the Neelon and Champagne (NEECHAM) Confusion Scale, have been validated for use by nonphysician healthcare professionals [22]. Yet, many health care professionals have difficulty recognizing delirium in elderly patients or do not use the screening tools available. Steis and Fick [23] found in a systematic review that even nurses who are able to define delirium may not recognize it in patients and may conduct only a basic mental status assessment. Similarly, a study of Armstrong and colleagues [24] found that physicians were likely to evaluate patients for delirium only once during hospitalization. Moreover, in another study, only $40 \%$ of participating physicians reported that they follow the recommendation of the Society of Critical Care Medicine to monitor routinely for delirium, e.g., by conducting assessments daily [9].

The utility of screening tools has been assessed in three systematic reviews (See Table 1). For example, Neto et al. [25] conducted a systematic review and meta-analysis of screening tools that could be used by nondelirium experts. Sixteen articles were included in the final analysis. All sixteen used the DSMD- IV criteria, with expert assessment as the standard. Study samples ranged in size from 29-181 subjects. Twelve of the sixteen evaluated the Confusion Assessment Method for the Intensive Care Unit (CAM-ICU). Three of the remaining four studies each evaluated one instrument: the Nursing Delirium Screening Scale (Nu-DESC), the Delirium Detection Score (DDS) and the Neelon and Champagne Confusion Scale. The prevalence of delirium ranged from $14 \%$ to $87 \%$, with the highest prevalence in a study with a high proportion of older patients ( $>65$ years). Only the CAM-ICU and $\mathrm{Nu}$-DESC were included in the meta-analysis. The CAMICU was reported to have high specificity and a high likelihood ratio for a positive score. Significant heterogeneity was reported among the CAM-ICU studies. The main finding in this meta-analysis was that the Nu-DESC had a higher sensitivity than CAM-ICU for diagnosis of delirium. The CAMICU showed high specificity, however, and for that reason it is useful to exclude a delirium diagnosis.

The CAM diagnostic algorithm is based on four elements: (1) an acute onset of mental status changes that fluctuate; (2) inattention; 3) disorganized thinking; and (4) an altered level of consciousness. The diagnosis of delirium is made if the patient evidences both (1) and (2) and either (3) or (4). A limitation of the CAM, however, is that the level of consciousness (LOC) must be assessed before a patient can be assessed for delirium. The Richmond Agitation Sedation Scale can be used to assess LOC and if the score is $\geq-3$, the Cam-ICU can be initiated.

The Nu-DESC is an observational, eight-item system that does not require patient cooperation. The assessment occurs over a 24-hour period of time versus the CAM-ICU, which is a measure of the state of the patient in a given moment of time. Different cut-off values were used in different studies. Four studies used the cutoff value of 4 and one the cutoff of 3 , making it difficult to pool results.

A systematic review of the Confusion Assessment Method (CAM) examined the psychometric properties, adaptations, translations, and application of this tool [26•]. A total of 260 primary studies with a total of 1,071 subjects were included in this review. Of these, ten articles were validation studies (examined performance characteristics, including sensitivity, specificity, and reliability), 16 evaluated adaptations (the tool was used in a different setting or to refine the diagnosis), 12 evaluated translations (foreign-language version), and 222 demonstrated applications of a variety of screening tools, including CAM (e.g., to rate delirium as a risk factor or outcome). In this latter group of studies, CAM was the most commonly used delirium-screening tool. Inter-rater reliability was moderate to high in all of the studies in this group. Results indicated an overall sensitivity of $95 \%$ and specificity of $89 \%$.

The ten validation studies included in the review reported sensitivities of $46 \%$ to $100 \%$ with three of the studies reporting sensitivities of $70 \%$ or less [26•]. Reasons for the low sensitivities were posited to be related to nurses or research assistants conducting the assessment without formal training in the use of the CAM as well as the use of the CAM with populations who had a high rate of dementia, depression, and psychosis which may make recognition of delirium more difficult. When formal cognitive testing such as the mini-mental status examination is used before delirium screening, the accuracy of the CAM rating increased.

One of the adaptations of CAM - the CAM-ICU - was developed specifically for the ventilated, nonverbal patient. When compared with ratings from experts using DSM-IV criteria, the CAM-ICU was reported to have sensitivity of $95 \%$ to $100 \%$, specificity of $93 \%$ to $98 \%$, and inter-rater reliability of 0.79 to 0.95 . CAM-rated delirium predicted poor functional outcomes, serious falls, mortality, adverse clinical and post-surgical outcomes, inadequate pain control, increased hospital stays, an increase in the use of sedatives or restraints, and institutionalization.

The CAM has also been incorporated into interventions that have been systematically evaluated. It has been used to test the efficacy of delirium practice guidelines by specialized delirium care teams for the recognition, management, and outcomes of delirious patients. The CAM is used nationally 
and internationally based on its favorable performance characteristics with reference standard ratings, e.g., the DSM-IV criteria, and has been included in more than 30 practice guidelines. It has also been used as a basis for an educational program on delirium recognition for nurses, and as part of routine screening for elderly ED patients [16].

Based on these findings, the authors of this systematic review [26•] make the following recommendations: (1) Optimize identification of delirium by training personnel on CAM. (2) In making cognitive assessments (e.g., with the MMSE or Short Portable Mental Status Questionnaire) include CAM as well. (3) To recognize delirium and avoid missing hypoactive, subtle, or atypical cases, use CAM in combination with clinical judgment and other formal screening measures.

Levy et al. [16] reviewed papers specifically related to delirium screening for hospital-based patients experiencing a stroke. In this review of 20 studies, it was determined that delirium occurs in $26 \%$ of patients who have a stroke ( $95 \%$ CI $19-33 \% ; p>.05$ ). The methods most commonly used to identify delirium were generic assessment tools such as the Delirium Rating Scale or the CAM or both. It was difficult to compare and group studies because of the wide variation in the way delirium was detected and the timing and frequency of delirium assessment. This has highlighted the importance of establishing delirium screening guidelines within stroke medicine for early identification.

In conclusion, while CAM and CAM-ICU are the most widely used screening tools, the CAM-ICU has higher sensitivity and specificity. In other words, the CAM and CAMICU tools are able to correctly identify both those patients with delirium (sensitivity) and those patients who are not experiencing delirium (sensitivity). Improving the ability of physicians and nurses to identify delirium will require educational interventions and is greatly needed.

\section{The Evidence on Preventive Strategies for Acute Delirium}

This section summarizes the evidence found to date for the effectiveness of several strategies to prevent the incidence of acute delirium and improve outcomes. Hempenius et al. [27] reviewed 16 systematic reviews to determine whether there is evidence that any intervention prevents delirium. Effectiveness was similar for pharmacological interventions, multi-component interventions, and single-strategy interventions (OR: 0.58 ; CI: $0.39-0.87$ versus OR: 0.59 ; CI: $0.38-0.92$ versus OR: 1.05 ; CI: $0.09-11.57$ ).

Summarized below are findings from two systematic reviews $[28 \bullet \bullet, 29]$ that focused on non-pharmacological interventions (See Table 1). The more recent one, by Milisen et al. $[28 \bullet \bullet]$, synthesized the findings of seven studies; four focused on interventions to prevent the development of delirium and three studies focused on early recognition and treatment of delirium. Of the four studies relating to prevention, one was a randomized control study, two were case-matched controlled and one was a controlled trial. The primary goal of the review was to identify the types and efficacy of multicomponent nonpharmacologic prevention strategies for delirium in hospitalized older adults. The review included a total of 1,248 medical and surgical patient aged 65 years and over who did not have a diagnosis of delirium based on the DSM criteria. The prevention strategies utilized in the studies included a variety of nonpharmacological interventions implemented by the healthcare team including specially trained volunteers, geriatric nurse specialists, geriatricians, social workers, occupational therapists and nutritionists. Interventions included pre-op consultation or consultation within 24 hours postoperatively, along with daily visits by a geriatrician or geriatric nurse specialist. Based on the daily evaluation, the geriatricians or geriatric nurse specialists made treatment recommendations relating to oxygen delivery, fluid/electrolyte balance, pain, medications, bowel/bladder function, nutrition, mobilization, and prevention, detection and treatment of complications, environment and treatment of agitation. Many of the recommended interventions were preventative in nature. Other preventative interventions included nursing staff education regarding patient orientation and communication, mobilization, environmental modifications, caregiver education, medication management and discharge planning. Standardized intervention protocols for patients who experienced cognitive impairment, sleep deprivation, immobility, visual and hearing impairment and/or dehydration was also evaluated.

Particularly noteworthy among the conclusions of this review was that promoting comfort, safety, hydration and oxygenation were most effective in reducing the incidence of delirium. For the patients in the seven studies reviewed by Milisen et al. [28••], delirium was prevented in one-third, and the incidence of severe delirium was lessened by over one-half. In a subgroup analysis, surgical patients without premorbid dementia or functional decline benefited most from any intervention, whereas multicomponent interventions were most effective for patients who exhibited one or two risk factors of delirium, specifically visual impairment, severe illness, cognitive impairment and high blood urea nitrogen to creatinine ratio.

An earlier systematic review by Cole and colleagues y found that proactive geriatric consultation within 24 hours of hip surgery reduced the incidence and severity of delirium, and that the preventive strategies studied worked better in a group of surgical patients as opposed to medical patients. Cole, et al.,, suggested that this might be due to the younger age and lesser severity of disease in the surgical patients.

In conclusion, there are few systematic reviews on the prevention of delirium. Those that are available indicate that the use of both pharmacologic and nonpharmacologic prevention strategies are successful, particularly when combined 
with identification of patents most at risk for delirium such as those with visual or hearing impairment or severe illness. Pharmacologic management may be beneficial, perhaps not as a first line approach, but instead at the discretion of the healthcare provider when the unique clinical conditions of the patient warrant its use.

\section{Conclusion}

Meaningful use of the findings generated by an overview of 13 systematic reviews related to the identification, screening and prevention of acute delirium in the hospitalized elderly can be made by incorporating the following best practices:

1. A tool, specifically a checklist, for physicians and nurses should be developed to stratify patients in different risk categories and to develop preventive strategies.

2. Meriperidine (Demerol), morphine and benzodiazapines should be avoided to the extent possible for those at elevated risk for delirium.

3. Although CAM was the most specific bedside tool for the assessment of delirium in critically ill patients, its low sensitivity in routine, daily practice limits its utility as a screening test. It should be used in conjunction with clinical judgment and other mental status examinations.

4. To make optimal use of CAM as a screening tool, physicians and nurses need to be trained on it.

6. All patients at risk for delirium should receive assessment.

7. Geriatric consultation within 24 hours before surgery can reduce the incidence and severity of delirium.

8. Promoting comfort, safety, hydration and oxygenation were most effective in reducing the incidence of delirium.

9. Importantly, assessment for delirium should be a routine part of daily practice, rather than a single event during a hospitalization.

Disclosure No potential conflicts of interest relevant to this article were reported.

\section{References}

Papers of particular interest, published recently, have been highlighted as:

- Of importance

•• Of major importance

1. Siddiqi N, House AO, Holmes JD. Occurrence and outcome of delirium in medical in-patients: a systematic literature review. Age Ageing. 2006;35(4):350-64.

2. Holly C, Cantwell ER, Jadotte Y. Actue delirium: differentiation and care. Crit Care Nurs Clin North Am. 2012;24(1):131-47.
3. Ely EW, Gautam S, Margolin R, et al. The impact of delirium in the intensive care unit on hospital length of stay. Intensive Care Med. 2001;27:1892-900.

4. Schuurmans MJ, Duursma SA, Shortridge-Baggett LM. Early recognition of delirium: review of the literature. J Clin Nurs. 2001;10(6):721-9.

5. American Psychiatric Association. Diagnostic and Statistical manual of Mental disorders. Fourthth ed. Washington DC: American Psychiatric Association; 2000.

6. Inouye SK. Delirium in older persons. N Engl J Med. 2006;354 (11):1157-65.

7. Rothschild JM, Leape LL. The nature and extent of medical injury in older patients: executive summary. Washington (DC): Public Policy Institute, AARP; 2000.

8. US Department of Health and Human Services. 2004 CMS statistics (CMS Publication No 03445). Washington, (DC): Centers for Medicare and Medicaid Services; 2004.

9. Ely EW, Shintani A, Truman B, et al. Delirium as a predictor of mortality in mechanically ventilated patients in the intensive care unit. JAMA. 2004;291:1753-62.

10. McAvay GJ, Van Ness PH, Bogardus ST, et al. Older adults discharged from the hospital with delirium: 1-year outcomes. J Am Geriatr Soc. 2006;54:1245-50.

11. Milbrandt EB, Deppen S, Harrison PL, et al. Costs associated with delirium in mechanically ventilated patients. Crit Care Med. 2004;32:955-62.

12. WHO. Health Topics: Risk Factors, para 1. Retrieved September $12,2012$.

13. Elie M, Cole M, Primeau F, Bellavance F. Delirium risk factors in elderly hospitalized patients. J Gen Intern Med. 2008;13:204-12.

14. •- Mattar Chan MF, Childs CI. Factors causing acute delirium in critically ill adult patients: a systematic review. JBI Library of Systematic Reviews. JBI000464. 2012;10(3):187-231. This paper presents the rigorous method necessary in a systematic review. The authors report that patients in different hospital settings exhibit different risk factors for delirium making patient assessment a complex process. For example, the presence of sepsis and heavy alcohol use were the most significant risk factors in the medical ICU, while in surgical ICU, high APACHE (Acute Physiology and Chronic Health Evaluation) II scores and high serum cortisol level influence the development of delirium.

15. Van Rompaey B, Schuurmansc MJ, Shortridge-Baggett LM, Truijenb S, Bossaerte L. Risk factors for intensive care delirium: a systematic review. Intensive Crit Care Nurs. 2008;24:98-107.

16. Levy CG, Mead GE, Nicol K, Rush R, van Wijck F. Delirium in acute stroke: screening tools, incidence rates and predictors: a systematic review. J Neurol. 2012;259:1590-9.

17. Koster S. Risk factors of delirium after cardiac surgery: a systematic review. Eur J Cardiovasc Nurs. 2011;10:197.

18. Fong HK, Sands L, Leung J. The role of postoperative analgesia in delirium and cognitive decline in elderly patients: a systematic review. Anesth Analg. 2006;102:1255-66.

19. Dasgupta M, Dumbrell AC, Maw J. Preoperative risk assessment for delirium after noncardiac surgery: a systematic review. J Am Geriatr Soc. 2006;54:1578-89.

20. Khan BA, Zawahiri M, Campbell NL, Fox GC, Weinstein EJ, Nazir A, et al. Delirium in hospitalized patients: implications of current evidence on clinical practice and future avenues for research-a systematic evidence review. J Hosp Med. 2012;7(7):580-9.

21. - Mason S, Storr AN, Ritcheia G. Post operative cognitive dysfunction and post operative delirium: a systematic review with MetaAnalysis. J Alzheimers Dis. 2010;22:S67-79. This metaanalysis demonstrated that rates of delirium are unlikely to be influenced by the route of anesthesia.

22. Devlin JW, Fong JJ, Howard EP, Skrobik Y, McCoy N, Yasuda C, et al. Assessment of delirium in the intensive care unit: nursing practices and perceptions. Am J Crit Care. 2008;17(6):555-65. 
23. Steis MR, Fick DM. Are nurses recognizing delirium? A systematic review. J Gerentol Nurs. 2008;34(9):40-8.

24. Armstrong SC, Cozza KL, Wantanable KS. The misdiagnosis of delirium. Psychosomatics. 1997;38:433-9.

25. Neto AS, Nassar Jr A, Cardoso S, Manetta J, Pereira V, Espósito D, et al. Delirium screening in critically ill patients: a systematic review and meta- analysis. Crit Care Med. 2012;40:1946-51.

26. - Wei LA, Wei BA, Fearing MA, Sternberg EJ, Inouye SK. The confusion assessment method: a systematic review of current usage. J Am Geriatr Soc. 2008;56:823-30. This review studied the psychometric properties of the Confusion Assessment Method, which is the most commonly used delirium screening tool. Results indicated an overall sensitivity of
$95 \%$ and specificity of $89 \%$. Inter-rater reliability was moderate to high in all studies.

27. Hempenius L, van Leeuwen B, van Asselt D, Hoekstra H, Wiggers T, Slaets J, et al. Structured analyses of interventions to prevent delirium. Int J Geriatr Psychiatry. 2011;26:441-50.

28. •• Milisen K, Lemiengre J, Braes T, Foreman M. Multi-component intervention strategies for managing delirium in hospitalized older people: systematic review. J Adv Nurs. 2005;52:79-90. This review demonstrates that basic aspects of care with attention to comfort, safety, hydration, and oxygenation proved to be the most effective strategies in preventing delirium.

29. Cole MG, Primeau F, McCusker J. Effectiveness of interventions to prevent delirium in hospitalized patients: a systematic review. CMAJ Can Med Assoc J. 1996;155(9):1263-8. 